INPLASY

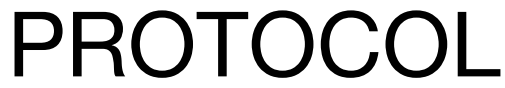

To cite: Wang et al. InstrumentAssisted Soft Tissue Mobilization for Increasing Range of Knee Motion: A protocol for systematic review and meta-analysis. Inplasy protocol 202220123. doi: 10.37766/inplasy2022.2.0123

Received: 28 February 2022

Published: 28 February 2022

Corresponding author: Yufeng Wang

wangchn@126.com

Author Affiliation:

Changchun University of Chinese Medicine.

Support: Y202044671.

Review Stage at time of this submission: Preliminary searches.

Conflicts of interest: None declared.

\section{Instrument-Assisted Soft Tissue Mobilization for Increasing Range of Knee Motion: A protocol for systematic review and meta-analysis}

\author{
Wang, Y1; Zhang, J2; Pang, T3; Liu, C4; Li, J5; Yao, J6.
}

Review question / Objective: Population: Patients with limited knee movement; Intervention: instrument-assisted soft tissue mobilization; Comparison: other therapy; Outcome: joint range of knee motion and Japanese Orthopedic Association (JOA) score.

Information sources: We will collect relevant articles by searching the following databases: PubMed, Web of Science, Scopus, Cochrane Library, Embase, China National Knowledge Infrastructure, China Science and Technology Journal Database and Wanfang Data. All databases will be searched from inception to December 30, 2021, by the following words: knee*, Femorotibia, Fascia*, Muscle Contraction, Muscle Strength, Muscle Fatigue, Muscle Tonus, degenerative joint disease, degenerative arthritis, Joint Range of Motion, Joint Flexibility, Range of Motion, Passive Range of Motion, Instrument-Assisted Soft Tissue Mobilization, IASTM*, Randomized controlled trial, Controlled clinical trial, RCT, etc.

INPLASY registration number: This protocol was registered with the International Platform of Registered Systematic Review and Meta-Analysis Protocols (INPLASY) on 28 February 2022 and was last updated on 28 February 2022 (registration number INPLASY202220123).

\section{INTRODUCTION}

Review question / Objective: Population: Patients with limited knee movement; Intervention: instrument-assisted soft tissue mobilization; Comparison: other therapy; Outcome: joint range of knee motion and Japanese Orthopedic Association (JOA) score.

Condition being studied: The knee joint is the largest and most complex flexor joint in the body, and knee disorders are common in clinical practice, among which knee stiffness and limitation of motion are a 
serious post-knee injury complication, commonly due to various causes, mainly long-term cast braking, scar adhesions, tissue contracture, heterotopic ossification of the knee joint, poor joint surface recovery, lack of standardized rehabilitation guidance, and patients' failure to actively engage in functional The majority of patients with post-traumatic knee stiffness have been found to have a stiff knee. Most patients with post-traumatic knee stiffness have a combination of quadriceps contracture, reduced elasticity, and reduced patellar movement, resulting in limited knee extension and flexion mobility, which seriously affects patients' daily life. There are various clinical treatments for post-traumatic knee stiffness, including manual release, incisional release under direct vision, minimally invasive arthroscopic release, rehabilitation manipulation, and instrument-assisted soft tissue mobilization (IASTM). Among them, IASTM is a soft tissue release procedure that detects and treats myofascial restrictions to improve mobility and reduce pain. IASTM has been reported to be effective in improving knee mobility and improving quality of life. After our preliminary study, we found that there is no systematic review on whether IASTM is safe and effective in patients with limited knee mobility. Therefore, we used this protocol for a comprehensive evaluation of the efficacy and safety of knee mobility.

\section{METHODS}

Participant or population: All patients with limited knee movement will be included without limitation of age, race, sex, economic level, and severity.

Intervention: All participants completed the same warm-up prior to the intervention. Controlled interventions included control groups with no treatment, sham/placebo groups, or other conventional treatments. The IASTM group received treatment on the gluteals/iliotibial band followed by the quadriceps, adductors, and hamstrings. Participants in both groups attended intervention sessions twice per week for 3 weeks. The interventions in the experimental group included only IASTM.

Comparator: Controlled interventions included control groups with no treatment, sham/placebo groups, or other conventional treatments.

Study designs to be included: Only randomized controlled trials (RCTs) will be included in this study.

Eligibility criteria: We will include only the literature of randomized controlled trials (RCTs) of aromatherapy massage for KOA. Nonrandomized controlled studies case reports, case series and reviews will not be included in this study.

Information sources: We will collect relevant articles by searching the following databases: PubMed, Web of Science, Scopus, Cochrane Library, Embase, China National Knowledge Infrastructure, China Science and Technology Journal Database and Wanfang Data. All databases will be searched from inception to December 30, 2021, by the following words: knee*, Femorotibia, Fascia*, Muscle Contraction, Muscle Strength, Muscle Fatigue, Muscle Tonus, degenerative joint disease, degenerative arthritis, Joint Range of Motion, Joint Flexibility, Range of Motion, Passive Range of Motion, InstrumentAssisted Soft Tissue Mobilization, IASTM*, Randomized controlled trial, Controlled clinical trial, RCT, etc.

Main outcome(s): The primary outcome was scales of the range of knee motion.

Additional outcome(s): Secondary endpoints included Visual analog scale, Symptom score, Lysholm knee scoring scale, Adverse events.

Quality assessment / Risk of bias analysis: The quality of all RCTs will be evaluated with the Cochrane Collaboration tool. Two authors (Junjie Yao and Jiahui Li) will independently conduct quality evaluations, and any controversy will be addressed by discussion with another author (Tingting Pang). 
Strategy of data synthesis: The metaanalysis of data from included outcomes will be performed using the RevMan V.5.4.1, and we will choose a randomized or fixed effect model for data statistics according to the results of the heterogeneity test. The enumeration data were expressed as relative risk (RR), and the weight mean difference (WMD) was used as the measurement data; each effect amount was expressed in $95 \%$ confidence interval (Cl). The specific methods were as follows: If the heterogeneity was low $\left(I^{2}<50 \%\right.$, the fixed-effects model was used for data synthesis. If there is high heterogeneity $\left(I^{2}>\right.$ $50 \%$ ), the random-effects model will be used for data synthesis after excluding possible heterogeneity sources. The investigation methods included subgroup and sensitivity analyses. If data cannot be synthesized, we provide a descriptive analysis to solve this problem.

Subgroup analysis: If there was high heterogeneity (12>50\%) among the included studies, we conducted a subgroup analysis to analyze the sources of heterogeneity according to the following factors: age, sex, race, courses, sample sizes, different methods of IASTM, and other possible factors affecting the results.

Sensitivity analysis: To test the stability and reliability of the results of this study, we conducted a sensitivity analysis according to the following points: method quality, sample size, and missing data. After that, we will perform a data analysis again and compare the results. If there was no directional change after the sensitivity analysis, the results were stable.

Country(ies) involved: China.

Keywords: Instrument-Assisted Soft Tissue Mobilization; Range of Knee Motion; Systematic review; Meta-analysis.

Contributions of each author:

Author 1 - Yufeng Wang.

Author 2 - Jiangchun Zhang.

Author 3 - Tingting Pang.

Author 4 - Chang Liu.

Author 5 - Jiahui Li.

Author 6 - Junjie Yao. 